\title{
CLASSIC GOUT IN AN ADOLESCENT WITH DOWN'S SYNDROME. A CASE REPORT
}

\begin{abstract}
Angela Luzia Branco Pinto Duarte ${ }^{1}$, Carolina Dias da Silva Amorim¹, Amália Maria Fernandes de Sá Duarte Filha ${ }^{1}$, Marco Felipe Macedo Alves ${ }^{1}$, Tânia Caroline Castro², Fernando Gurgel ${ }^{3}$, Rafaela Silva Guimaraes Goncalves ${ }^{1, \star}$
\end{abstract}

1.Universidade Federal de Pernambuco, Recife (PE), Brazil; 2.Universidade Cidade de São Paulo, São Paulo (SP), Brazil; 3.Centro Diagnóstico Lucilo Ávila, Recife (PE), Brazil.

*Corresponding author: rafa_sgg@hotmail.com

\section{BACKGROUND}

Several reports have indicated higher prevalence of hyperuricemia in patients with Down' syndrome (DS), but gout has been rare. Our objective is to describe an adolescent who was diagnosed with traumatic arthritis and rheumatic fever for four years due to recurrent acute mono/oligo arthritis, whose final diagnosis was gout.

\section{CASE REPORT}

In June 2021, a 20-year-old male presented arthritis of the right elbow for three days. His crisis started a week ago with intense pain and swelling in the left foot which spontaneously improved. On the fourth day his right elbow was very swollen and painful, and prednisolone was recommended. At the age of 16 (2017), he had his first episode with elbow arthritis, which fully recovered within seven days without medication. In 2019 he had arthritis in his left foot. At that time, he went to the orthopedic emergency and his foot was immobilized for a week. In November 2020 he had arthritis in the right wrist followed by involvement of the right elbow. Prednisolone was prescribed with resolution of the crisis in 10 days. He also has shown Hashimoto's thyroiditis and congenital heart disease - total atrioventricular septal defect, moderate atrioventricular valve reflux and mild subvalvular pulmonary stenosis. He has used synthroid, furosemide, digoxin, captopril and aldactone. On examination, the patient had arthritis of the right elbow and left hallux. Laboratory evaluation was remarkable for hyperuricemia since 2019 not previously valued. Ultrasound of the right elbow showed moderate joint effusion in the coronoid fossa associated with mild synovial thickening, irregularity of the capitellum cartilage with echogenic foci on its surface, suggesting crystal deposition (double contour) with signs of crystal deposition. Ultrasound of the left hallux showed irregularity of the surfaces of the metatarsophalangeal joint, with synovial thickening, erosion on the medial surface of the metatarsal epiphysis, where there are echogenic foci suggesting crystal deposits. There are also signs of double contour (podagra). Digital X-ray of the feet revealed erosion and cystic lesions in the hallux.

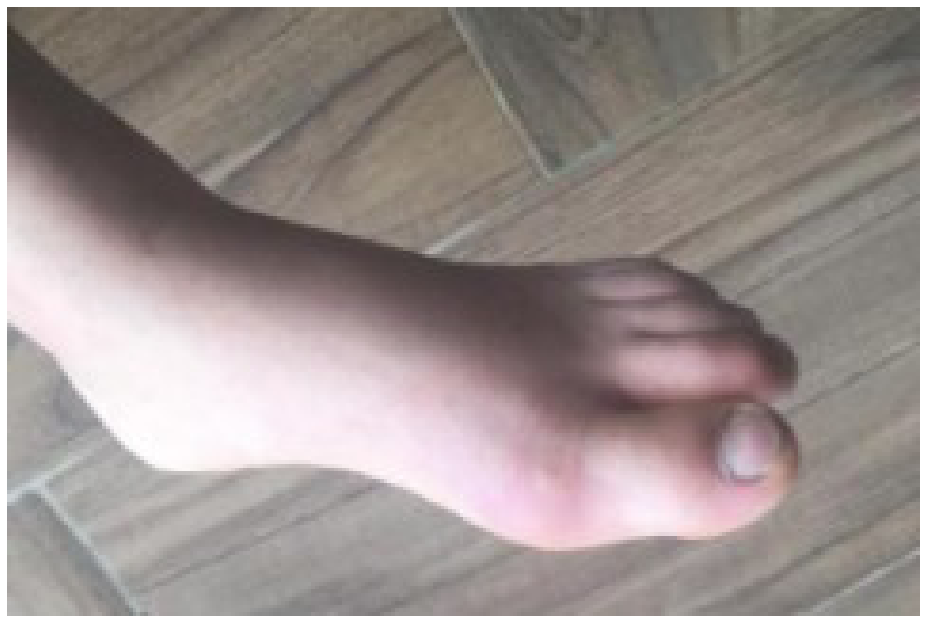

Figure 1. In 2017 - left hallux crisis. Patient file. 


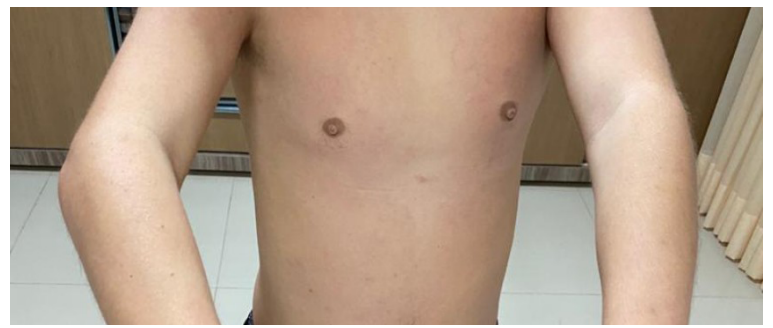

Figure 2. Right elbow arthritis, with painful extension block.

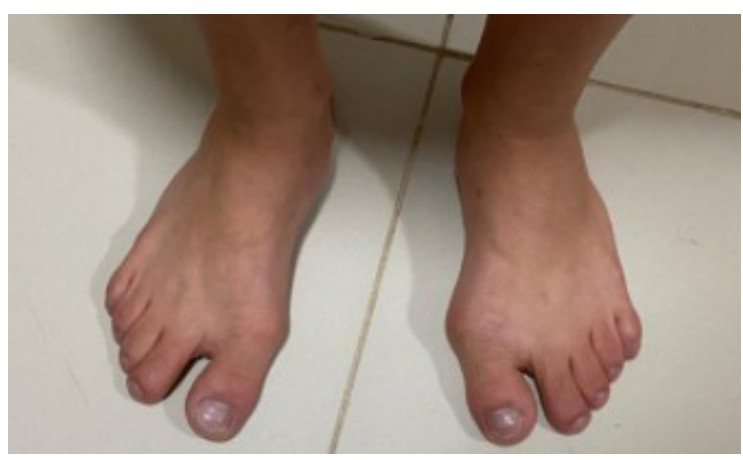

Figure 3. Cyanosis fingers. Increased left hallux volume.
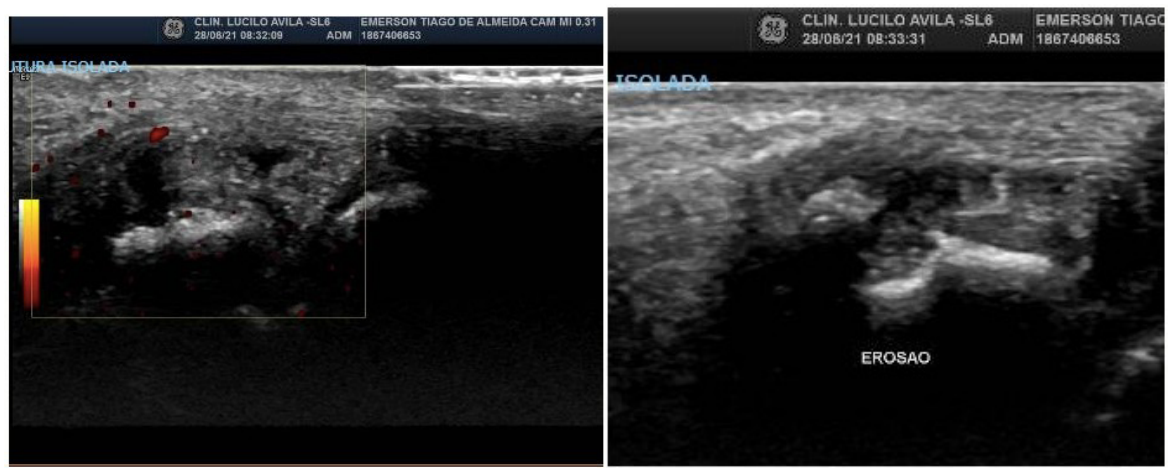

Figure 4. USG right elbow - Arthropathy with signs of mild synovitis and osteochondral lesion in the capitellum with signs of crystal deposition.
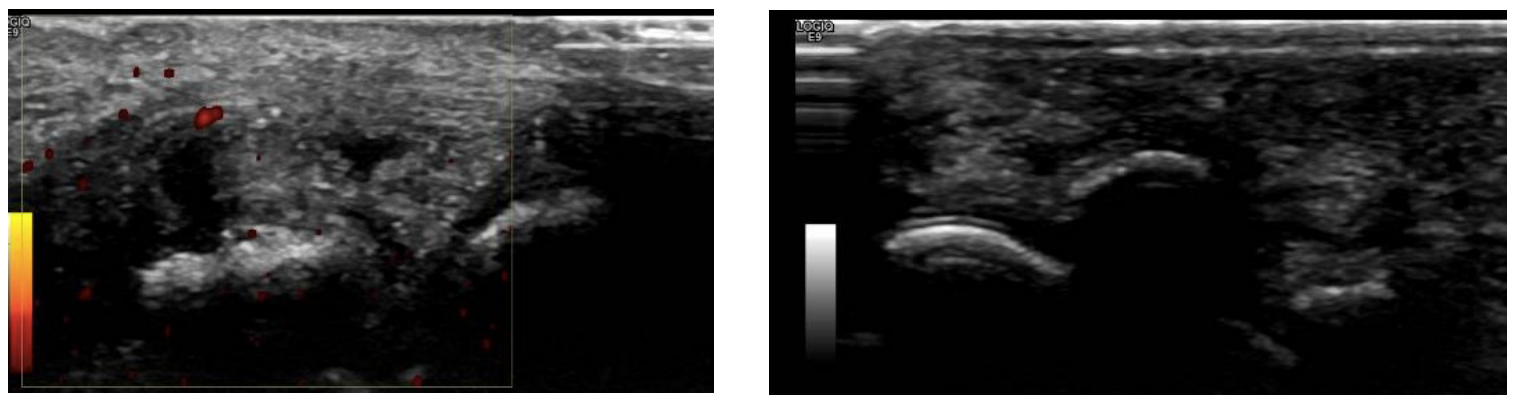

Figure 5. USG left hallux - Metatarsophalangeal arthropathy with signs of synovitis, crystal deposition and double contour sign. 

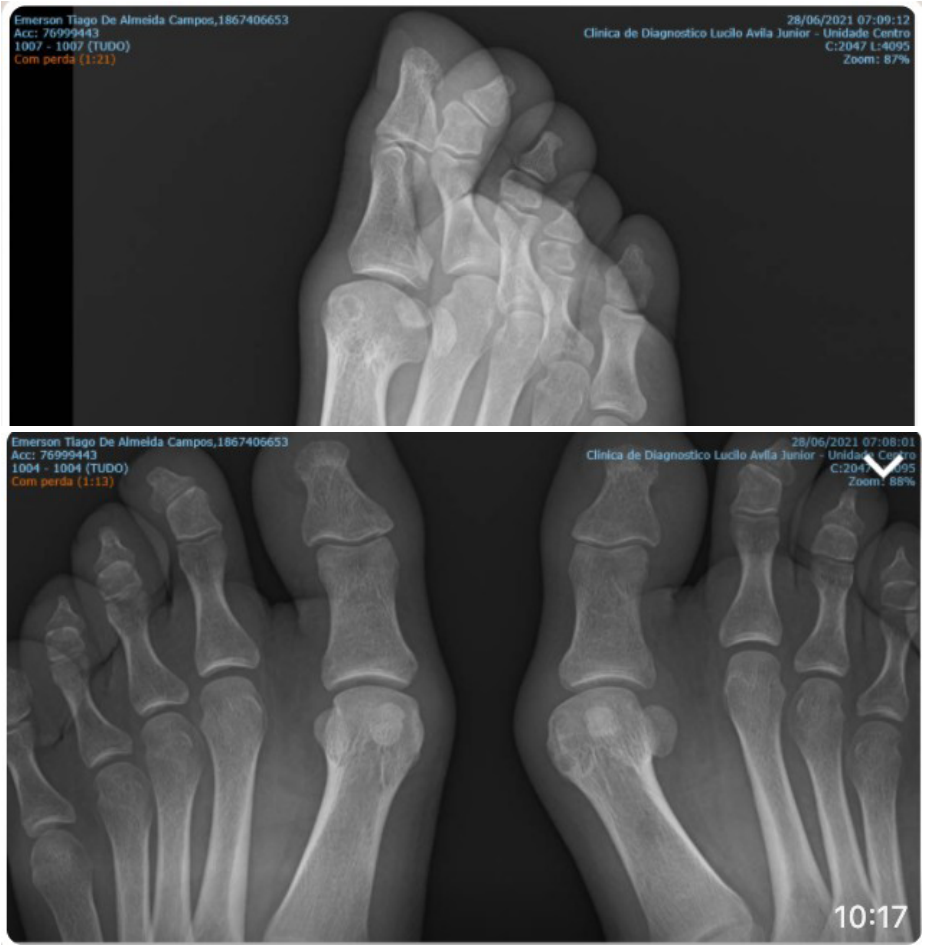

Figure 6. Digital X-ray of the feet - erosion and cystic lesions in the hallux.

\section{CONCLUSION}

Hyperuricemia has frequently been reported in DS, although only few cases develop gout. Our patient had congenital heart disease and polycythemia as additional risk factors. It's important to remember the possibility of gout in patients with DS who present recurrent acute arthritis.

\section{KEYWORDS}

Down' syndrome, Hyperuricemia, Gout. 